\title{
Molding with nanoparticle-based one-dimensional photonic crystals: a route to flexible and transferable Bragg mirrors of high dielectric contrast $\dagger$
}

\author{
Received 3rd February 2009, Accepted 8th April 2009 \\ First published as an Advance Article on the web 22nd April 2009 \\ DOI: 10.1039/b902090j
}

Mauricio E. Calvo, Olalla Sánchez Sobrado, Gabriel Lozano and Hernán Míguez*

\begin{abstract}
Self-standing, flexible Bragg mirror films of high refractive index contrast and showing intense and wide Bragg peaks are herein presented. Nanoparticle-based one-dimensional photonic crystals are used as templates to infiltrate a polymer, which provides the multilayer with mechanical stability while preserving the dielectric contrast existing in the mold. Such films can be lifted off the substrate and used to coat another surface of arbitrary shape.
\end{abstract}

Materials that combine flexibility and high reflectivity at certain wavelength ranges can be used to protect substrates of arbitrary shape from a specific radiation, or to provide them with the desired color. When this effect has a structural origin, i.e., it is due to the periodic variation of the refractive index, ${ }^{1}$ then heating due to absorption is also prevented. In spite of their interest, there exist few examples of such materials operating in the visible and near infrared. This is due to the difficulty of devising and realizing materials possessing simultaneously a periodic modulation of the refractive index at the submicron length scale and a mechanically stable flexible structure. In recent years, some important advances in this direction have been made by using different materials chemistry approaches. Flexible lamellar structures with one-dimensional photonic crystal properties were developed by self-assembly of a mixture of copolymers and homopolymers. ${ }^{2}$ Alternating regions of polyisoprene (refractive index $n=1.51)$ and polystyrene $(n=1.59)$ gave rise to the periodic dielectric structure. Polymer layers were also used combined with inorganic materials to make flexible mirrors in the infrared region. ${ }^{3,4}$ In another case, the porous lattice of polystyrene artificial opals is infiltrated with an elastomer (PDMS, $\mathrm{n}=1.43$ ) to build a socalled "photonic paper", a highly reflecting sheet whose color may vary selectively when put in contact with the appropriate ink. ${ }^{5}$ Based on this achievement, flexible polymer colloidal crystal lasers with light emitting planar defects have been developed. ${ }^{6}$ Also, three-dimensional self-organization of polystyrene microspheres within a hydrogel matrix $(1.33<\mathrm{n}<1.6)$ has been used to attain flexible colored mirrors with glucose sensing properties. ${ }^{7,8}$ Also, melt compression of all latex core-shell particles gives rise to three-dimensionally ordered arrays of the polystyrene cores in a matrix made of flexible polyethylacrylate $(\mathrm{n}=1.41) .{ }^{9}$ In most of these examples, color arises as a result of the built-up periodicity of two different organic materials whose refractive index contrast is low. This implies a limitation in the range of frequencies that can be reflected by the mirror, since the

Instituto de Ciencia de Materiales de Sevilla, Consejo Superior de Investigaciones Cientificas (CSIC), Américo Vespucio 49, 41092 Sevilla, Spain.E-mail: hernan@icmse.csic.es

$\dagger$ Electronic supplementary information (ESI) available: Experimental details. See DOI: 10.1039/b902090j photonic stop band width in terms of frequency is directly proportional to the refractive index contrast between the two layers. ${ }^{10}$ Besides, low contrast also implies that the intensity of the Bragg reflectance peak will also be small for a reduced number of layers.

Herein we present a route to attain flexible Bragg mirrors of high dielectric contrast through molding with nanoparticle-based one-dimensional photonic crystals. ${ }^{11}$ These porous nanostructures are infiltrated with a polymer compound, providing them with enough mechanical stability to be lifted off and transferred to another surface of arbitrary shape. The high dielectric contrast existing between the $\mathrm{SiO}_{2}$ and $\mathrm{TiO}_{2}$ layers within the photonic crystal template is preserved after infiltration of the void volume with a polymer. Thus, the resulting flexible film presents wide Bragg reflectance peaks $(\Delta \lambda / \lambda \approx 40 \%$, where $\Delta \lambda$ is the full width at half maximum) of high intensity $\left(I_{\max } \approx 70 \%\right)$. Its spectral position can be precisely tuned by changing the lattice parameter of the original mold. Also, we demonstrate that different flexible photonic crystals can be piled up to attain optical coatings that efficiently block wide spectral ranges. Finally, we demonstrate that this method combined with thermal annealing allows one to transfer purely inorganic photonic crystal films from one substrate to another while keeping a high optical quality.

One-dimensional photonic crystals (1DPC) herein used as templates are obtained by a bottom-up approach based on spincoating suspensions of $\mathrm{TiO}_{2}$ and $\mathrm{SiO}_{2}$ nanoparticles onto flat substrates, as has been thoroughly described in ref. 9. Such a multilayer structure displays bright structural color and possesses porosity that is controllable through both the degree of aggregation and the particle size of the precursor suspensions. ${ }^{12}$ In addition spin-coating deposition allows multilayer films to be obtained with high reproducibility and uniformity as was demonstrated recently by our group. $^{13}$

Preliminary studies on the environmental response of these materials already demonstrated their great potential as base materials for gas and liquid detection, ${ }^{14}$ as well as enhancers of the solar-to-electric power conversion efficiency of dye solar cells. ${ }^{15}$ In these cases, such 1DPCs were heated at $450{ }^{\circ} \mathrm{C}$ to sinter the nanoparticles they are made of, hence providing them with mechanical stability while keeping their porosity intact. For the purpose pursued in this work, we infiltrate the as-grown nanoparticle multilayers with a solution of polycarbonate. In order to do so, drops of such a solution are deposited and uniformly spread onto the 1DPC film. Once the solution has penetrated the interstices of the multilayer, the substrate is rotated using a spin-coater. By doing so, the excess liquid is removed from the film surface and the solution is evenly distributed within the porous multilayer. In the final stages of the spin-coating process, the organic solvent is evaporated from the interstitial sites, which are then partially filled with polycarbonate. This polymer also 
forms a layer a few microns thick on the film. The infiltrated and coated 1DPC is then mechanically stable and can be lifted off the substrate without losing its optical properties. In Fig. 1, we draw a scheme of the steps taken to infiltrate, lift off and transfer to a different substrate the photonic crystal multilayer films.

In Fig. 2, we show scanning electron microscope images of the cross-section of a 1DPC infiltrated with polycarbonate and separated from the substrate. It can be clearly seen in Fig. 2(a) that the order is preserved in the lifted off film. We observe that polycarbonate accumulates in the form of thin layers at the interfaces between the $\mathrm{SiO}_{2}$ and $\mathrm{TiO}_{2}$ nanoparticle layers, but only when the $\mathrm{SiO}_{2}$ layer was deposited on top of $\mathrm{TiO}_{2}$ one, as shown in the higher magnification image shown in Fig. 2(b). This indicates that the vertical downwards convection of the precursor solution induced by the rotation of the substrate is hindered in the compact upper surface of the $\mathrm{TiO}_{2}$ layers. Concurrent evaporation of the organic solvent leaves behind thin layers of polycarbonate deposited in the vicinity of that interface. In contrast, the solution already within the $\mathrm{TiO}_{2}$ layers can easily flow towards the $\mathrm{SiO}_{2}$ layers, which possess much larger pores. As will be demonstrated below, this implies that the polycarbonate will condensate mainly in the $\mathrm{SiO}_{2}$ layers, the polymer load of the $\mathrm{TiO}_{2}$ ones being much lower. In a closer look, as that shown in Fig. 2(b), the nanometer-sized polycarbonate threads holding the self-standing multilayer structure together can also be detected.

The infiltrated multilayers present enough mechanical stability to be lifted off the substrates they were deposited upon. This yields flexible Bragg mirrors that can be transferred to coat another surface. In Fig. 3, we show a series of pictures corresponding to the process of lifting off and transferring of such flexible photonic crystals. In Fig. 3(a) and (b) we show different polycarbonate-filled nanoparticlebased 1DPC of different lattice parameters deposited onto different types of substrates, namely glass (a) and silicon wafers (b). In Fig. 3(c), an infiltrated nanoparticle-based 1DPC is lifted off the glass substrate it was deposited on. The flexibility and highly colored reflection of the film can be readily seen in this photograph. Once separated, it can be transferred to a silicon wafer, as shown in Fig. 3(d), keeping its high reflectance intact. These films can be shaped in different forms and stick to surfaces of arbitrary curvature. This is illustrated in Fig. 3(e) and (f), in which we show a couple of flexible 1DPC films circularly cut and one of such films in conformal contact with the surface of a steel sphere. It can be appreciated how the blue Bragg mirror adapts to the curved surface maintaining its high reflectance.

The spectroscopic analysis of the reflected light shows that the high dielectric contrast existing in the original template is preserved in the self-standing film. In Fig. 4(a), the specular reflectance spectra of a nanoparticle-based 1DPC film before and after being infiltrated with polycarbonate are shown.

An intense (90\% reflectance for stacks made of 12 alternate layers) and wide Bragg reflection peak is observed for the original template, which is a direct consequence of the large refractive index contrast between the $\mathrm{SiO}_{2}$ and $\mathrm{TiO}_{2}$ nanoparticle layers. After infiltration, the Bragg peak intensity slightly decreases ( $80 \%$ reflectance) and its position shifts towards longer wavelengths, which indicates that the void interstices of the template are being filled with polycarbonate and also that it causes the refractive index contrast to diminish a little.

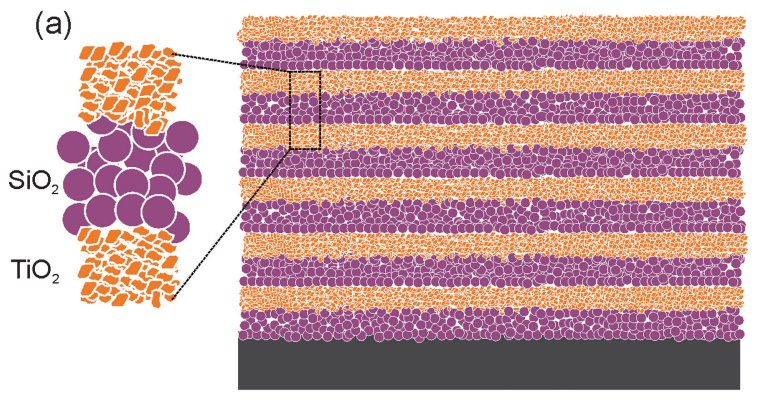

(c)

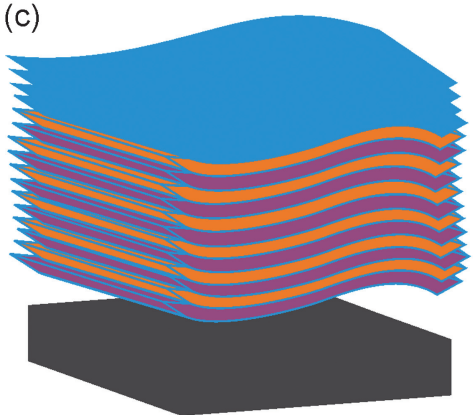

(d)

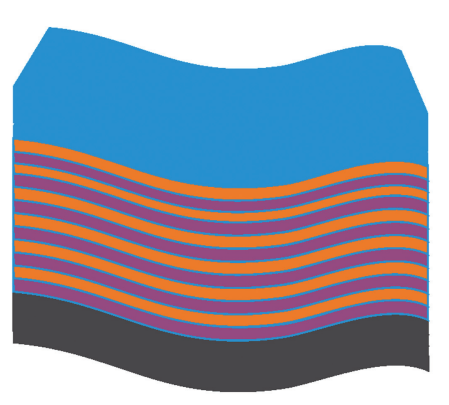

(b)

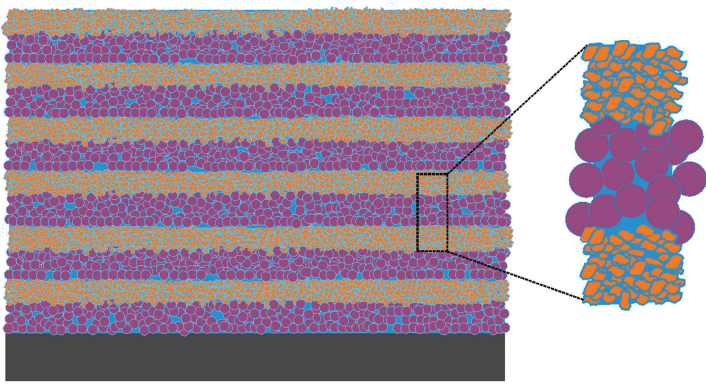

(e)

Fig. 1 Scheme of the preparation of flexible and transferable Bragg mirrors. (a) Cross-section of a porous 1DPC film, formed by six alternating layers of $\mathrm{SiO}_{2}$ nanoparticles (violet circles) and $\mathrm{TiO}_{2}$ nanocrystals (orange polygons) deposited onto a flat substrate. The detail shows the interpenetration of the $\mathrm{TiO}_{2}$ layer into the $\mathrm{SiO}_{2}$ layer and their porous structure. (b) A 1DPC film infiltrated with polycarbonate. The inset shows the interstitial space filled with the polymer. (c) Simplified representation $\left(\mathrm{SiO}_{2}\right.$ layers, violet stacks; $\mathrm{TiO}_{2}$ layers, orange stacks) of the mechanical separation of the infiltrated multilayer from the substrate, which yields a flexible and self-supported Bragg mirror. Polycarbonate can be found both into the voids of the multilayer and deposited onto its outmost surface. (d) Self-supported 1DPC film can be put in conformal contact with a surface of arbitrary shape. (e) Finally, the 1DPC may be heated to remove the polymer and recover the original structure of the purely inorganic template. 

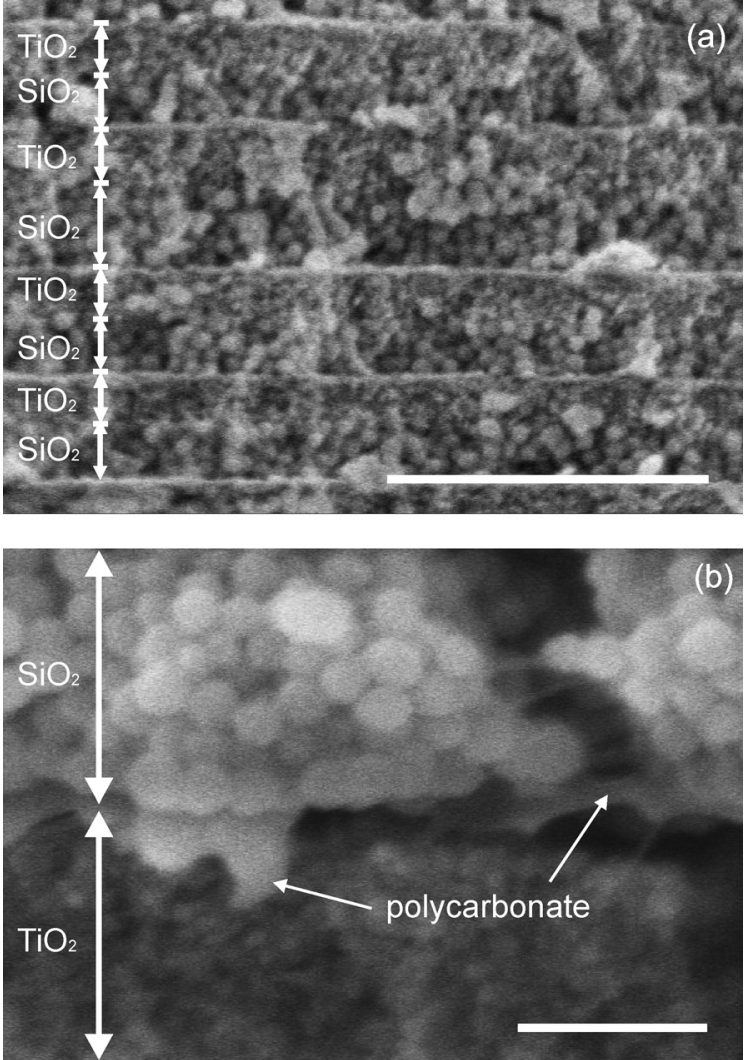

Fig. 2 Field emission scanning electron microscopy images of a nanoparticle based 1DPC film infiltrated with poly(bisphenol-A-carbonate). (a) Cross-section of a polymer infiltrated twelve layer film deposited on flat glass (scale bar $=500 \mathrm{~nm}$ ). (b) Local structural fracture in the $\mathrm{SiO}_{2}$ layer that allows observation of the polycarbonate threads connecting the nanoparticles as well as a detail of the accumulation layer between upper silica and lower titania layers (scale bar $=100 \mathrm{~nm}$ ).

In fact, by fitting the optical reflectance spectra using a scalar wave approximation, ${ }^{16,17}$ we estimated the refractive indices of the asgrown $\mathrm{TiO}_{2}$ and $\mathrm{SiO}_{2}$ nanoparticle-based films in the template to be $n_{\mathrm{TiO} 2}=1.70$ and $n_{\mathrm{SiO} 2}=1.24$ respectively. This implies that the pore volume fraction in the films, as calculated using the Brugemann equation, ${ }^{18}$ are $48 \%$ and $43 \%$ respectively, assuming that bulk $\mathrm{TiO}_{2}$ and $\mathrm{SiO}_{2}$ have refractive indices of 2.44 and 1.425 respectively. After infiltration, the indices of the alternate layers are $n_{\mathrm{TiO} 2}=1.74$ and $n_{\mathrm{SiO} 2}=1.36$, which yields pore volume fractions of $45 \%$ and $15 \%$ respectively, hence indicating that $\mathrm{SiO}_{2}$ layers are much more effectively infiltrated than $\mathrm{TiO}_{2}$ ones. These estimations, extracted from the analysis of the optical response, are in good agreement with the SEM observations and support the mechanism of infiltration and hindered diffusion proposed above. In Fig. 4(b), the reflectance spectra of several flexible 1DPC films measured after being transferred from the original substrates to new ones are plotted. In these examples, different lattice parameters were achieved by varying the rotation speed of the substrate from one photonic crystal to the other.

These results demonstrate that, after being lifted off and transferred, the films still behave as efficient (70\% reflectance) Bragg mirrors of wide band gap $(\Delta \lambda \lambda \approx 40 \%)$ whose reflectance maximum can be tailored to measure to match any visible or near infrared range just by changing the lattice parameter of the periodic structure used as
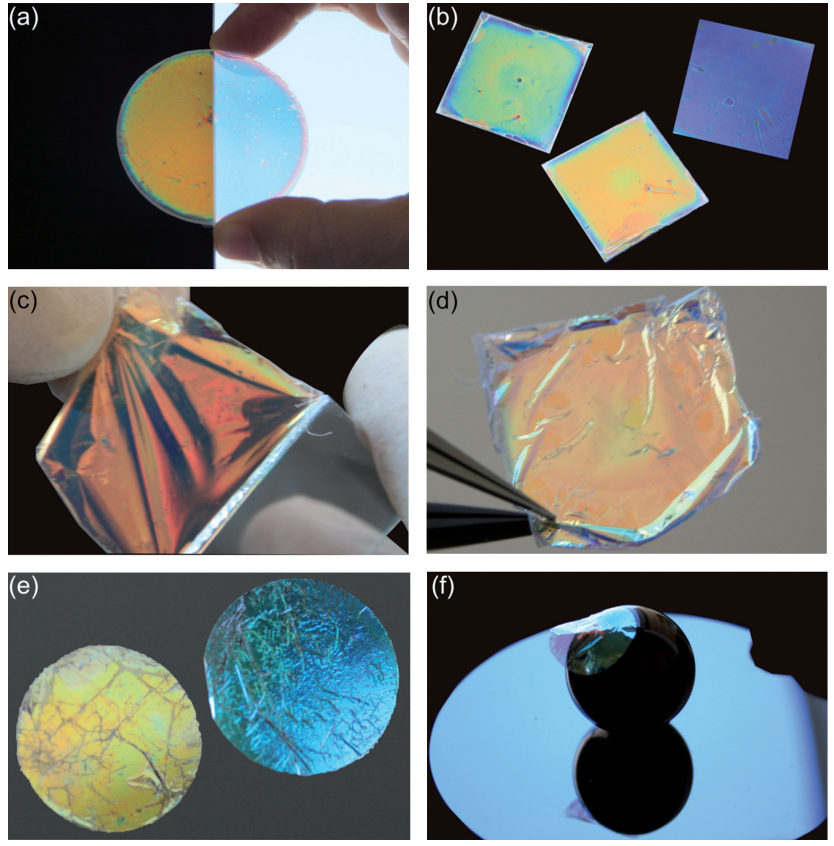

Fig. 3 Photographs of some Bragg mirrors. (a) A twelve layer nanoparticle based 1DPC deposited on a circular glass and infiltrated with polycarbonate. One half of the photonic crystal coated glass is exposed to daylight from the back and the other is placed onto a black background to observe the different transmission and reflection optical responses. (b) 1DPC of different lattice parameters deposited on different square Si and glass substrates and infiltrated with polycarbonate. (c) A picture taken while peeling off a polycarbonate infiltrated 1DPC previously deposited on glass. The flexibility of the resulting film can be readily appreciated. (d) The flexible Bragg mirror shown in (c) being put in conformal contact with the flat surface of a Si wafer. (e) Different round cuts of flexible Bragg mirrors of different lattice parameters deposited on Si wafers. (f) A circularly cut $1 \mathrm{DPC}$ film being put in conformal contact with a black painted sphere.

template. Such flexible 1DPCs stick to one another easily and can be readily piled up to attain tandem Bragg mirrors that are able to block wider spectral ranges. A scheme of such a structure is given in Fig. 5(a). The reflectance spectrum of a flexible tandem Bragg mirror is plotted in Fig. 5(b), where it can be clearly seen that a wider reflectance maximum (see dashed line) results from the stacking of two flexible 1DPC of different lattice parameters. The spectra of the individual flexible Bragg mirrors forming the tandem structure measured separately are also shown (solid lines).

The flexible structures herein presented can also be used as a means to coat another surface with a purely inorganic photonic crystal. After being lifted off and stuck to a new surface, the polymer guest can be removed and the structure of the original template recovered by thermal annealing $\left(450{ }^{\circ} \mathrm{C}\right.$ for 30 minutes). Fig. 6(a) shows an SEM picture of a transferred one-dimensional photonic crystal after removal of the polycarbonate. Closer analysis reveals that, in this example, the multilayer has been re-deposited upside down with respect to the original template, as the observed interpenetration of lower $\mathrm{TiO}_{2}$ layers on upper $\mathrm{SiO}_{2}$ ones indicates. After all this processing, the final structure still shows a clear reflectance maximum $(50 \%)$ and its porosity remains intact, as the optical analysis shows. In Fig. 6(b), the reflectance spectra of a transferred infiltrated nanoparticle based Bragg mirror before (dotted line) and after (solid line) 

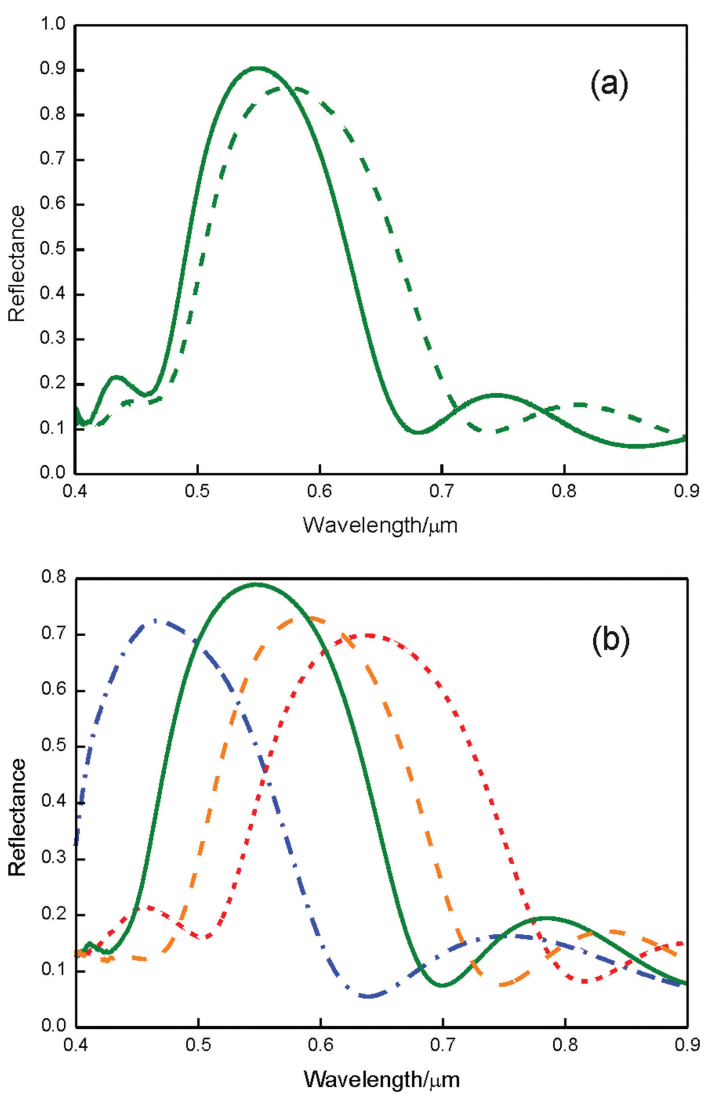

Fig. 4 (a) Specular reflectance spectra obtained from a ten layer nanoparticle-based 1DPC before (solid line) and after (dashed line) infiltration with polycarbonate. (b) Specular reflectance spectra of flexible Bragg mirrors re-deposited on flat glass. Each flexible photonic crystal whose optical response is herein shown presents a different lattice parameter. This variation was achieved using the same substrate rotation speed $(\omega=$ $6000 \mathrm{rpm})$ to deposit the original templates but changing either the acceleration $\operatorname{ramp}(\gamma)$ or the nanoparticle concentration. In each case: $\left[\mathrm{TiO}_{2}\right]=4 \mathrm{wt} . \%,\left[\mathrm{SiO}_{2}\right]=2 \mathrm{wt} . \%, \gamma=8450 \mathrm{rpm} \mathrm{s}^{-1}$ (dash-dotted line) $\gamma$ $=5850$ (solid line); $\gamma=4550$ (long-dashed line); and $\left[\mathrm{TiO}_{2}\right]=4 \mathrm{wt} . \%$, $\left[\mathrm{SiO}_{2}\right]=2.5 \mathrm{wt} . \%, \gamma=5850$ (short-dashed line). (a)

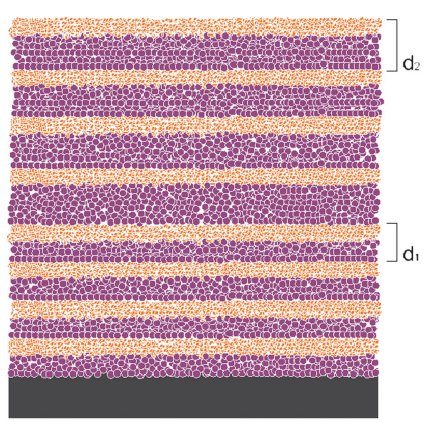

(b)

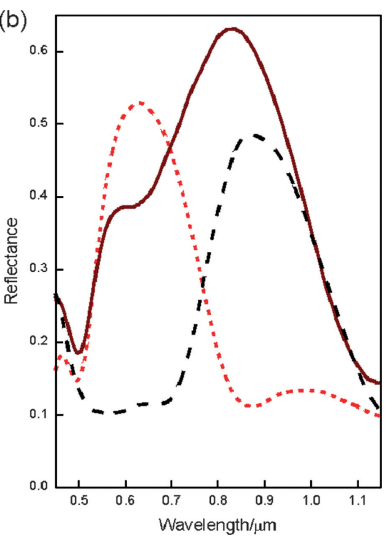

Fig. 5 (a) Scheme of a tandem multilayer, where $d_{1}$ and $d_{2}$ represent the unit cell constants of each 1DPC present in the stack. (b) Specular reflectance spectra obtained for two flexible eight layer 1DPC with different cell parameters measured separately (short and long dashed lines) and after being piled up to form a hybrid stack (solid line).
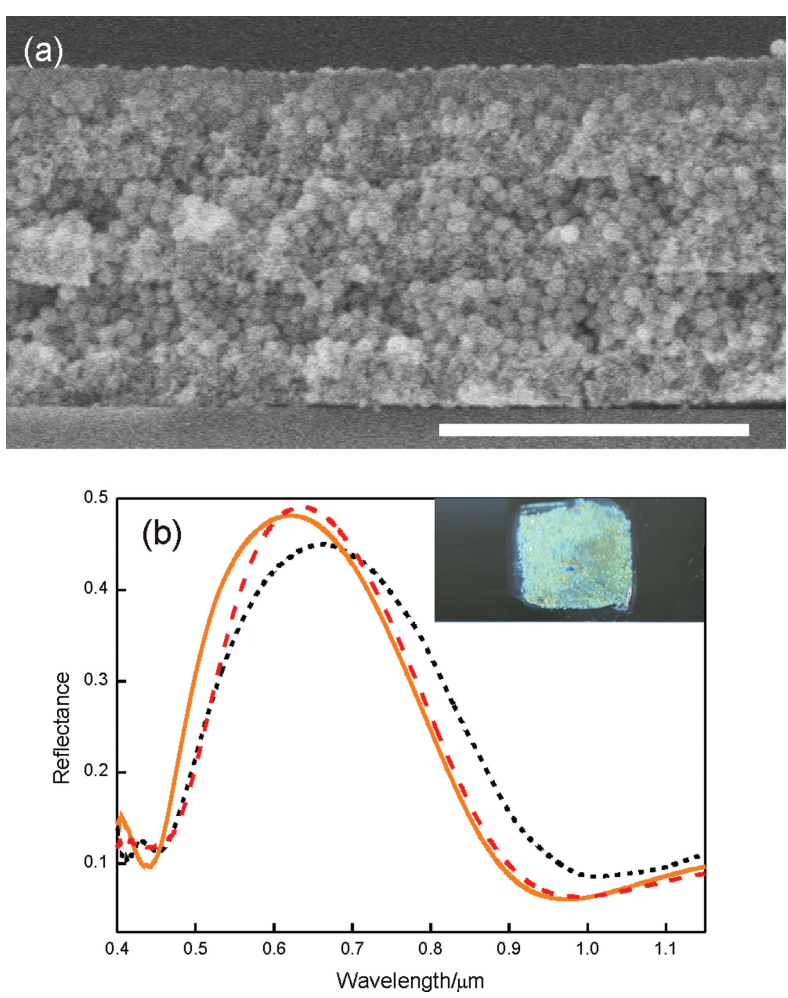

Fig. 6 (a) FESEM image of a cross-section of a transferred Bragg mirror after being annealed at $450{ }^{\circ} \mathrm{C}$ for 30 minutes (scale bar $=500 \mathrm{~nm}$ ). (b) Specular reflectance spectra of a transferred Bragg mirror re-deposited on flat borosilicate glass before (short dotted line) and after (solid line) removal of the polycarbonate by thermal annealing. The reflectance spectrum of the same nanoparticle multilayer after being infiltrated with ethanol is also plotted (dashed line).

thermal annealing are displayed. The removal of polycarbonate decreases the effective refractive index of the structure and gives rise to a blue shift of the Bragg peak position $(\Delta \lambda \approx 50 \mathrm{~nm})$.

The spectrum of the same structure after being soaked in ethanol shows in turn a clear red shift of the Bragg peak (dashed line, $\Delta \lambda \approx$ $20 \mathrm{~nm}$ ), which demonstrates that the porosity is preserved and it is still accessible after annealing. Similar environmental response has been thoroughly illustrated for the original templates in refs. 11 and 12. The inset in Fig. 6(b) shows a photograph of a transferred and annealed Bragg mirror.

In conclusion, we have demonstrated a new method to attain flexible Bragg mirrors in which the refractive index contrast is similar to that existing in supported films made of inorganic materials. We have proven that nanoparticle-based one-dimensional photonic crystals are suitable templates to infiltrate polymers. This provides them with the required mechanical stability to be lifted off and transferred, while keeping the high dielectric contrast existing in the original inorganic matrix. Although polycarbonate was chosen as the infiltrating polymer due to its ease of handling and the wide range of applications it finds nowadays, the method herein presented is generic and may be extended to any other polymeric compound, such as conducting polymers or elastomers. We believe it will open the route towards multifunctional flexible films showing a tailored optical response. Also, this method combined with thermal annealing provides a means to transfer purely inorganic Bragg mirrors from one 
substrate to another while retaining the structural and optical features of the original photonic matrix.

\section{Acknowledgements}

This work has been funded by the Spanish Ministry of Science and Education under grants MAT2008-02166, the Junta de Andalucia under project FQM-3579, and project Consolider HOPE CSD200700007.

\section{Notes and references}

1 H. A. Macleod, Thin Film Optical Filters, $3^{\text {rd }}$ Edition, Institute of Physics Publishing, London, 2001.

2 A. Urbas, R. Sharp, Y. Fink, E. L. Thomas, M. Xenidou and L. J. Fetters, Advanced Materials, 2000, 12, 812.

3 T. Druffel, N. Mandzy, M. Sunkara and E. Grulke, Small, 2008, 4, 459.

4 R. G. DeCorby, N. Ponnampalam, H. T. Nguyen and T. J. Clement, Advanced Materials, 2007, 19, 193.
5 H. Fudouzi and Y. Xia, Advanced Materials, 2003, 15, 892; H. Fudouzi and Y. Xia, Langmuir, 2003, 19, 9653.

6 S. Furumi, H. Fudouzi, H. T. Miyazaki and Y. Sakka, Advanced Materials, 2007, 19, 2067.

7 J. H. Holtz and S. A. Asher, Nature, 1997, 389, 829.

8 V. L. Alexeev, S. Das, D. N. Finegold and S. A. Asher, Clinical Chemistry, 2004, 50, 2353.

9 T. Ruhl, P. Spahn and G. P. Hellmann, Polymer, 2003, 44, 7625.

10 A. Yariv, P. Yeh, Optical Waves in Crystals, John Wiley and Sons, Inc., Hoboken, New Jersey, 2003.

11 S. Colodrero, M. Ocaña and H. Miguez, Langmuir, 2008, 24, 44304434.

12 M. E. Calvo, S. Colodrero, T. C. Rojas, M. Ocaña, J. A. Anta and H. Míguez, Adv. Func. Mater., 2008, 18, 2708.

13 M. E. Calvo, O. Sánchez-Sobrado, S. Colodrero and H. Míguez, Langmuir, 2009, 25, 2443-2449.

14 S. Colodrero, M. Ocaña, A. R. González-Elipe and H. Míguez, Langmuir, 2008, 24, 9135-9139.

15 S. Colodrero, A. Mihi, L. Häggman, M. Ocaña, G. Boschloo, A. Hagfeldt and H. Míguez, Advanced Materials, 2009, 21, 764-770.

16 K. W. K. Shung and Y. C. Tsai, Phys. Rev. B, 1993, 48, 11265.

17 A. Mihi and H. Míguez, J. Phys. Chem. B, 2005, 109, 15968.

18 H. C. Van de Hulst, Light Scattering by Small Particles, Dover Publications, New York, 1981. 